\title{
Enhancement of the retrapping current of superconducting microbridges of finite length
}

\author{
D. Y. Vodolazov ${ }^{1, *}$ and F. M. Peeters ${ }^{2}$ \\ ${ }^{1}$ Institute for Physics of Microstructures, Russian Academy of Sciences, 603950, Nizhny Novgorod, GSP-105, Russia \\ ${ }^{2}$ Departement Fysica, Universiteit Antwerpen (CGB), Groenenborgerlaan 171, B-2020 Antwerpen, Belgium \\ (Received 29 September 2011; revised manuscript received 23 December 2011; published 5 January 2012)
}

\begin{abstract}
We theoretically find that the resistance of a superconducting microbridge or nanowire decreases while the retrapping current $I_{r}$ for the transition to the superconducting state increases when one suppresses the magnitude of the order parameter $|\Delta|$ in the attached superconducting leads. This effect is a consequence of the increased energy interval for diffusion of the "hot" nonequilibrium quasiparticles (induced by the oscillations of $|\Delta|$ in the center of the microbridge) to the leads. The effect is absent in short microbridges (with length less than the coherence length) and it is relatively weak in long microbridges (with length larger than the inelastic relaxation length of the nonequilibrium distribution function). A nonmonotonous dependence of $I_{r}$ on the length of the microbridge is predicted. Our results are important for the explanation of the enhancement of the critical current and the appearance of negative magnetoresistance observed in many recent experiments on superconducting microbridges or nanowires.
\end{abstract}

DOI: 10.1103/PhysRevB.85.024508

PACS number(s): 74.25.Op, 74.20.De, 73.23.-b

\section{INTRODUCTION}

Recently, several experimental groups observed a negative magnetoresistance (NMR) of superconducting nanowires or microbridges at temperatures lower than the critical temperature. ${ }^{1-7}$ In Refs. 3-6 the authors demonstrated that in their case the effect is connected with the suppression of superconductivity in the bulk superconductor caused by the applied magnetic field. Moreover in Refs. 2-6 it was shown that this NMR appears together with an enhancement of the critical current ${ }^{8}$ of the nanowires in weak magnetic fields.

Presently there exist several theories ${ }^{10-14}$ which propose different mechanisms for the NMR. Reference 10 claims that it is connected with the suppression of a new channel of dissipation in superconducting wires by an applied magnetic field, while Refs. 11 and 12 argue that the suppression of the intrinsic pair-breaking resulting from the total spin-flip + non-spin-flip rate is responsible for the effect. In Ref. 13 the stabilization of the superconducting phase due to magneticfield-induced normal metal-superconductor boundaries at the ends of the microbridge was offered as the main mechanism for the negative magnetoresistance, while in Refs. 1,14, and 15 the effect was explained as due to a decrease of the charge imbalance decay length $\lambda_{Q}$ in weak magnetic fields.

Taking into account the strong dependence of both the NMR and the enhancement of $I_{c}$ on the length of the superconducting microbridge or nanowire (i.e., the effect does not exist in relatively long and short samples; see Refs. 3-6) we argue that the proposed mechanisms as put forward in Refs. 10-13 might not always be relevant for these experiments. Indeed their applicability is not limited by the length of the superconductors. Due to the same reason a decrease of $\lambda_{Q}{ }^{1,14,15}$ cannot explain the length dependence of the effect because it should lead to NMR in samples of arbitrary length. Besides, at currents close to the depairing current, $\lambda_{Q}$ starts to depend on the current ${ }^{16}$ and it smears its dependence on $H$. As a result this mechanism cannot explain the increase of $I_{c}$ in weak magnetic fields.
In Ref. 14 another mechanism for the enhancement of $I_{c}$ was proposed connected with the complete suppression of the order parameter in the superconducting leads. But this mechanism is not able to explain the monotonous enhancement of $I_{c}$ in weak magnetic fields (see Refs. 2, 5, and 6) when the superconducting leads are still in the superconducting state with a weakly suppressed order parameter.

Here, we offer an alternative mechanism that leads to the negative magnetoresistance of microbridges or nanowires and the enhancement of the critical (retrapping) current. Our explanation is based on the following idea: Even a weak suppression of the order parameter in the leads opens new energy channels for the diffusion of the "hot" quasiparticles from the microbridge where they are induced by oscillations of the order parameter. ${ }^{17}$ As a result the effective "temperature" of the quasiparticles in the microbridge decreases and the retrapping current for the transition to the superconducting state increases. Simultaneously it leads to decrease of the resistance of the microbridge at fixed current. Our proposed mechanism leads to no effect in very short and very long microbridges or nanowires (in agreement with the experiments ${ }^{3-6}$ ). Besides, we find that the retrapping current is a nonmonotonous function of the length of the microbridge.

The paper is organized as follows. In Sec. II we present the theoretical model. The current-voltage $(I-V)$ characteristics of microbridges of different length are presented in Sec. III for different values of the order parameter in the leads. In Sec. IV we discuss our result and in Sec. V we present our conclusions.

\section{MODEL}

To study the resistive state of the microbridge we use the set of equations (time-dependent Ginzburg-Landau equation for the superconducting order parameter coupled with the kinetic equations for the quasiparticle distribution function and the Usadel equations for the Green's functions) derived for "dirty" superconductors near the critical temperature of 
the superconductor: ${ }^{18-21}$

$$
\begin{aligned}
& N_{1} \frac{\partial \delta f_{L}}{\partial t}-D \nabla\left[\left(N_{1}^{2}-R_{2}^{2}\right) \nabla \delta f_{L}\right] \\
& =D \nabla\left(j_{\epsilon} f_{T}\right)-\frac{N_{1}}{\tau_{\text {in }}} \delta f_{L}-R_{2} \frac{\partial f_{L}^{0}}{\partial \epsilon} \frac{\partial|\Delta|}{\partial t}, \\
& \frac{\partial}{\partial t} N_{1}\left(f_{T}+e \varphi \frac{\partial f_{L}^{0}}{\partial \epsilon}\right)-D \nabla\left[\left(N_{1}^{2}+N_{2}^{2}\right) \nabla f_{T}\right] \\
& =D \nabla\left(j_{\epsilon} \delta f_{L}\right)-\frac{N_{1}}{\tau_{\text {in }}}\left(f_{T}+e \varphi \frac{\partial f_{L}^{0}}{\partial \epsilon}\right) \\
& -N_{2}|\Delta|\left(2 f_{T}+\hbar \frac{\partial f_{L}^{0}}{\partial \epsilon} \frac{\partial \phi}{\partial t}\right) \\
& \hbar D\left(\frac{d^{2} \Theta}{d x^{2}}+\frac{d^{2} \Theta}{d y^{2}}\right)+\left[\left(2 i \epsilon-\frac{\hbar}{\tau_{\text {in }}}\right)-\hbar D Q^{2} \cos \Theta\right] \sin \Theta \\
& +2|\Delta| \cos \Theta=0 \text {, } \\
& \frac{\pi \hbar}{8 k_{B} T_{c}} \frac{\partial \Delta}{\partial t}-\left(\Phi_{1}+i \Phi_{2}\right) \Delta \\
& =\xi_{G L}^{2}\left(\nabla-i \frac{2 e A}{\hbar c}\right)^{2} \Delta+\left(1-\frac{T}{T_{c}}-\frac{|\Delta|^{2}}{\Delta_{G L}^{2}}\right) \Delta .
\end{aligned}
$$

Here $\Delta=|\Delta| e^{i \phi}$ is a complex order parameter, $\xi_{G L}^{2}=$ $\pi \hbar D / 8 k_{B} T_{c}$ and $\Delta_{G L}^{2}=8 \pi^{2}\left(k_{B} T_{c}\right)^{2} / 7 \zeta(3)$ are the zerotemperature Ginzburg-Landau coherence length and the order parameter correspondingly. $Q=(\partial \phi / \partial x-2 e A / \hbar c)$ is a quantity which is proportional to the superfluid velocity $\left(v_{s}=\right.$ $D Q$ ) with $A$ the vector potential taken in the Landau gauge, $\varphi$ is the electrostatic potential, and $f_{L}(\epsilon)=f_{L}^{0}(\epsilon)+\delta f_{L}(\epsilon)$ is the longitudinal and $f_{T}(\epsilon)$ is the transverse part of the quasiparticle distribution function $2 f(\epsilon)=1-f_{L}(\epsilon)-f_{T}(\epsilon)$ [in equilib$\left.\operatorname{rium} f_{L}=f_{L}^{0}(\epsilon)=\tanh \left(\epsilon / 2 k_{B} T\right), f_{T}^{0}(\epsilon)=0\right] . N_{1}, N_{2}, R_{2}$ are the spectral functions which are determined by the Usadel equation for the normal $\alpha(\epsilon)=\cos \Theta=N_{1}(\epsilon)+i R_{1}(\epsilon)$ and anomalous $\beta_{1}=\beta e^{i \phi}, \beta_{2}=\beta e^{-i \phi}\left[\beta(\epsilon)=\sin \Theta=N_{2}(\epsilon)+\right.$ $i R_{2}(\epsilon)$ ] Green's functions.

Nonequilibrium corrections to the quasiparticle distribution function enters Eq. (3) via the potentials $\Phi_{1}=$ $\int_{0}^{\infty} R_{2} \delta f_{L} d \epsilon /|\Delta|$ and $\Phi_{2}=\int_{0}^{\infty} N_{2} f_{T} d \epsilon /|\Delta|$. Equations (1a) and (1b) are coupled due to the finite spectral supercurrent $j_{\epsilon}=\operatorname{Re}\left(\beta_{1} \nabla \beta_{2}-\beta_{2} \nabla \beta_{1}\right) / 2=2 N_{2} R_{2} Q$.

The advantage of Eqs. (1)-(3) in comparison with the ordinary or the extended ${ }^{20}$ time-dependent Ginzburg-Landau equations is that they allow us to take into account nonlocal effects. Here, under nonlocality we mean that if in one place of the superconductor and in some moment in time the quasiparticle distribution function $f(\epsilon)$ becomes nonequilibrium (due to some kind of perturbation) then the nonequilibrium correction to $f(\epsilon)$ will be nonzero over a distance $\sim L_{\text {in }}=\left(D \tau_{\text {in }}\right)^{1 / 2}$ around that point and during a time $\sim \tau_{\text {in }}$ after turning off the perturbation.

Before solving Eqs. (1)-(3) numerically we calculate the order parameter in a model 2D system (see Fig. 1) in the stationary state in the presence of an applied magnetic field. This result demonstrates the suppression of $|\Delta|$ in the leads by increasing $H$ and the weak influence on $|\Delta|$ in the microbridge. It also shows that instead of the $2 \mathrm{D}$ model we may use a $1 \mathrm{D}$ model where the suppression of the order parameter in (a)
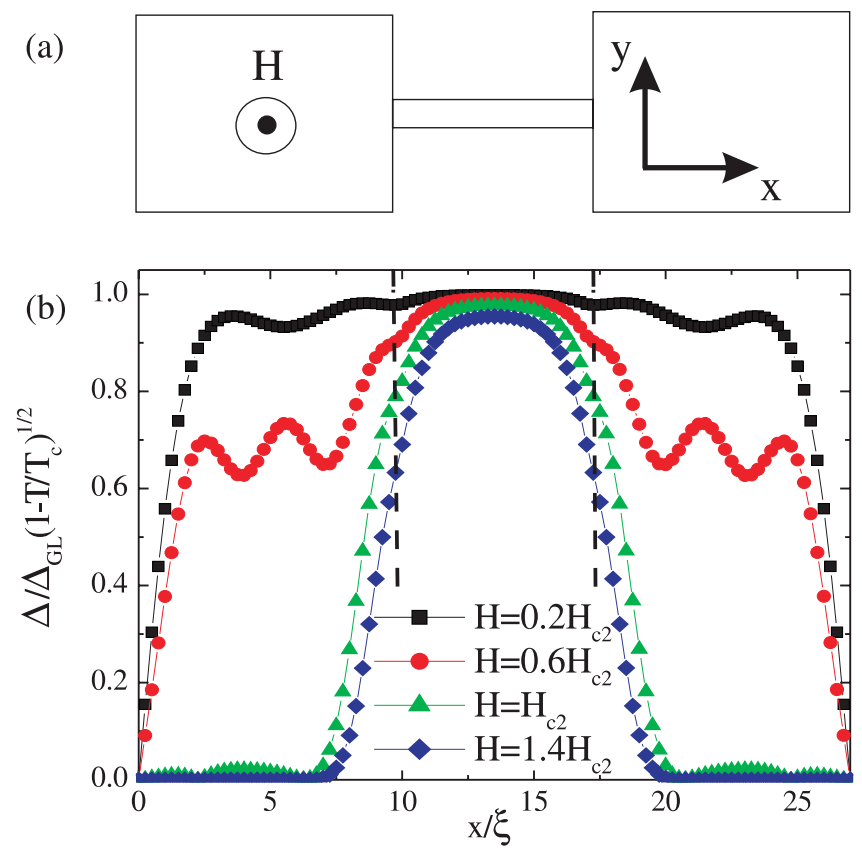

(c)
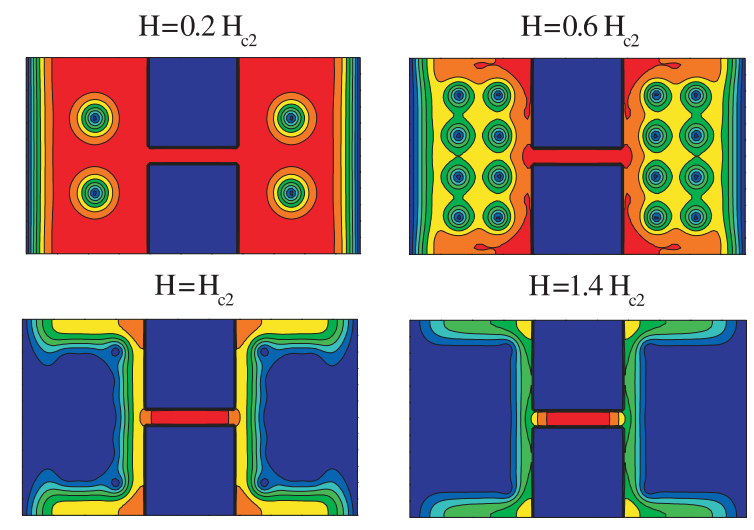

FIG. 1. (Color online) (a) Schematic illustration of the finitewidth Dayem microbridge connected with wide superconducting leads. (b) Dependence of the magnitude of the order parameter along the center line of the microbridge for different magnetic fields. (c) Contour plot of $|\Delta|$ in the leads and microbridge at different $H$ [blue (red) color corresponds to low (high) value for $|\Delta|$ ]. The length of the full system (leads + microbridge) is $27 \xi$, width $15 \xi$; length of microbridge is $7 \xi$ and width of microbridge is $\xi$.

the superconducting leads could be simulated by introducing locally a lower critical temperature. Thus we may use the model of Ref. 17 where we introduce a different critical temperature in the leads (see Fig. 2). The correlation between $T_{c}^{\prime}$ and $H$ is clear: Smaller $T_{c}^{\prime}$ corresponds to larger $H$.

The self-consistent set of Eqs. (1)-(3) was solved numerically using the method and boundary conditions presented in Ref. 17. Further, we use the following dimensionless units. The order parameter is scaled by $\Delta_{0}\left(\Delta_{0}=1.76 k_{B} T_{c}\right)$, distance is in units of the zero-temperature coherence length $\xi_{0}=\sqrt{\hbar D / \Delta_{0}}$, time in units of $t_{0}=\hbar / \Delta_{0}$, and temperature in units of the critical temperature $T_{c}$. The current is scaled in units of $j_{0}=\Delta_{0} \sigma_{n} /\left(\xi_{0} e\right)$ and the electrostatic potential is in units of $\varphi_{0}=\Delta_{0} / e$. It is useful to introduce the dimensionless inelastic relaxation time $\tilde{\tau}_{\text {in }}=\tau_{\text {in }} / t_{0}$ which is the main control 


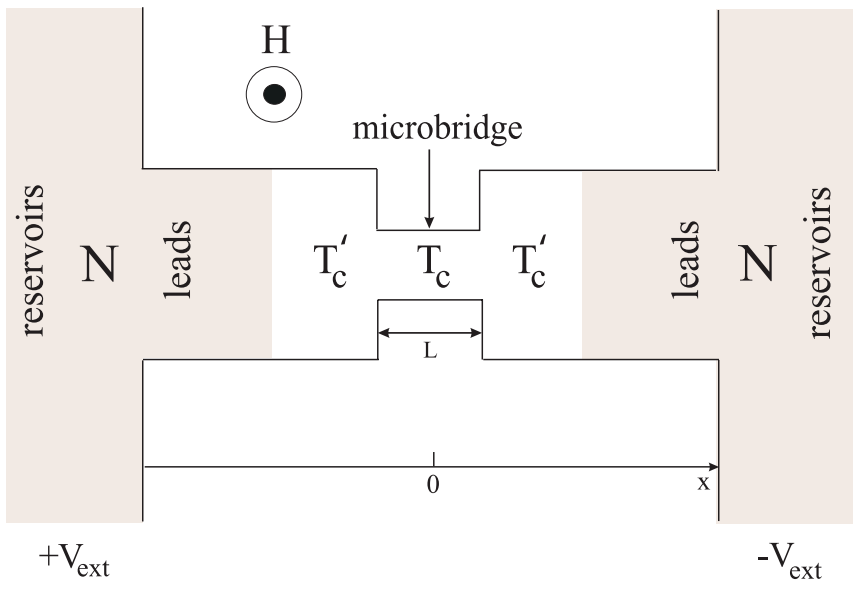

FIG. 2. (Color online) Schematic illustration of the model system. Critical temperature in the leads is smaller than the critical temperature of the microbridge.

parameter in the model described by Eqs. (1)-(3). For example in $\mathrm{MgB}_{2} \tilde{\tau}_{\text {in }} \simeq 4,{ }^{22}$ in $\mathrm{Nb}$ and $\mathrm{Pb} \tilde{\tau}_{\text {in }} \simeq 40$, in Sn $\tilde{\tau}_{\text {in }} \simeq 70$, in Al $\tilde{\tau}_{\text {in }} \simeq 3500$, and in $\mathrm{Zn} \tilde{\tau}_{\text {in }} \simeq 2 \times 10^{4} .^{16}$

\section{RESULTS}

In Fig. 3 we present the $I-V$ characteristics of the superconducting microbridge with length $L=21 \xi_{0}$ calculated for different values of the order parameter in the leads ${ }^{26}$ (in the inset we show distribution of the time-averaged order parameter in the microbridge and leads at $I=0.75 I_{c}$ ). Notice that the retrapping current increases and the resistance of the microbridge decreases when the order parameter is slightly suppressed in the leads. We should stress here that the critical current $I_{c}$ of the microbridge (at which the superconducting state becomes unstable) monotonically decreases with decreasing $T_{c}^{\prime}$.

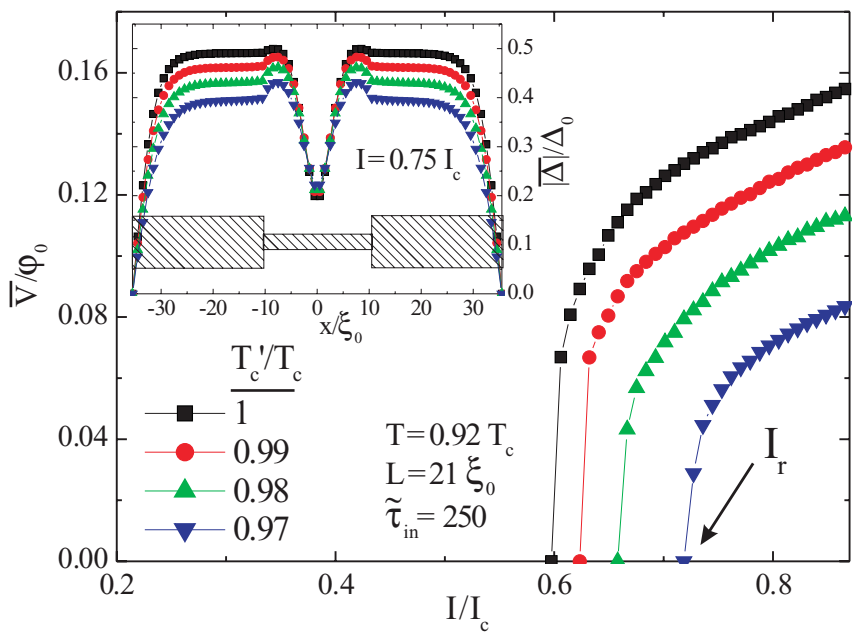

FIG. 3. (Color online) Current-voltage characteristics of the superconducting microbridge with length $L=21 \xi_{0} \simeq 4.9 \xi(T)$ calculated at $T=0.92 T_{c}$ and $\tilde{\tau}_{\text {in }}=250$. The inset shows the distribution of the time-averaged order parameter in the microbridge at different $T_{c}^{\prime}$. Current is normalized in units of the critical current of the microbridge with $T_{c}^{\prime}=T_{c}$.

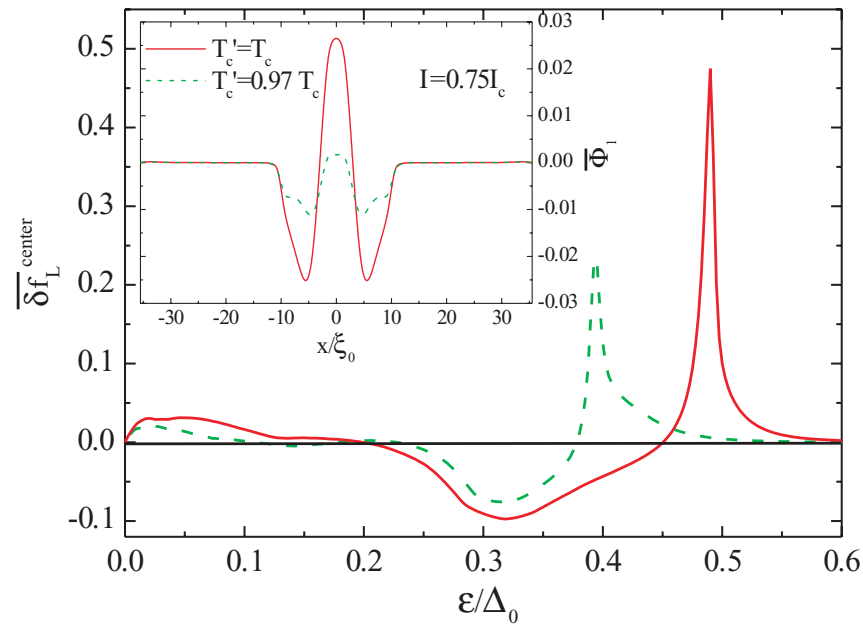

FIG. 4. (Color online) Energy dependence of the time-averaged $\overline{\delta f_{L}}$ in the center of the microbridge with length $L=21 \xi_{0} \simeq 4.9 \xi(T)$, $\tilde{\tau}_{\text {in }}=250$, and $T=0.92 T_{c}$ calculated for $I=0.75 I_{c}$. Solid and dashed curves corresponds to two different effective temperatures $T_{c}^{\prime}=T_{c}$ and $T_{c}^{\prime}=0.97 T_{c}$ in the leads, respectively. In the insert we present the spatial dependence of the time-averaged potential $\overline{\Phi_{1}}$ in the bridge with chosen parameters.

Our result can be explained as due to the enhanced diffusion of the "hot" quasiparticles induced by oscillations of the order parameter in the center of the microbridge ${ }^{17}$ when $\Delta_{\text {lead }}$ becomes smaller. Indeed, in this case the energy barrier connected with the finite energy gap at $\epsilon<|\Delta|_{\text {lead }}$ decreases and nonequilibrium quasiparticles in the wider energy interval can diffuse to the leads. Here we should remind the reader that the local energy gap in the microbridge is smaller than the local value of the order parameter due to the finite supercurrent and the spatial variation of $|\Delta| .{ }^{16,23}$ Therefore, "hot" quasiparticles may diffuse in the energy interval $\epsilon>|\Delta|_{\text {lead }}$ even when the local $|\Delta|$ in the microbridge is larger than $|\Delta|_{\text {lead }}$ (see inset in Fig. 3).

In order to illustrate the above effect we show in Fig. 4 the energy dependence of the nonequilibrium correction to $f_{L}$ in the center of the microbridge averaged over one period of Josephson oscillations. In the inset to Fig. 4 we present the spatial dependence of the time-averaged potential $\overline{\Phi_{1}}$ which corresponds to the effective "temperature" $T_{\text {eff }}=T+T_{c} \Phi_{1}$ of quasiparticles in the microbridge. ${ }^{17}$ One can easily see that with decreasing $\Delta_{\text {lead }}$ the energy interval where the quasiparticles are "heated" (corresponds to a negative sign of $\delta f_{L}$ ) decreases and it results in drastic changes of $\overline{\Phi_{1}}$.

The found effect depends strongly on the length of the microbridge. When $L \lesssim 2 \xi(T)$ the value of $\Delta_{\text {lead }}$ has the strongest effect on the value of $I_{r}$ (which itself is close to the critical current of the microbridge $I_{c}$; see Fig. 5) while the effect of "heating" is relatively weak (see Ref. 24). With decreasing $\Delta_{\text {lead }}$ both $I_{c}$ and $I_{r}$ monotonically decrease and the resistance increases; see Fig. 5. The critical length $L_{c}$ for which $I_{r}$ starts to increase depends on the inelastic relaxation time: the larger $\tilde{\tau}_{\text {in }}$ the shorter $L_{c}$. For example $11 \xi_{0}<L_{c}<15 \xi_{0}$ for $\tilde{\tau}_{\text {in }}=250$ while $17 \xi_{0}<L_{c}<$ $21 \xi_{0}$ for $\tilde{\tau}_{\text {in }}=60$ at $T=0.92 T_{c}$. 


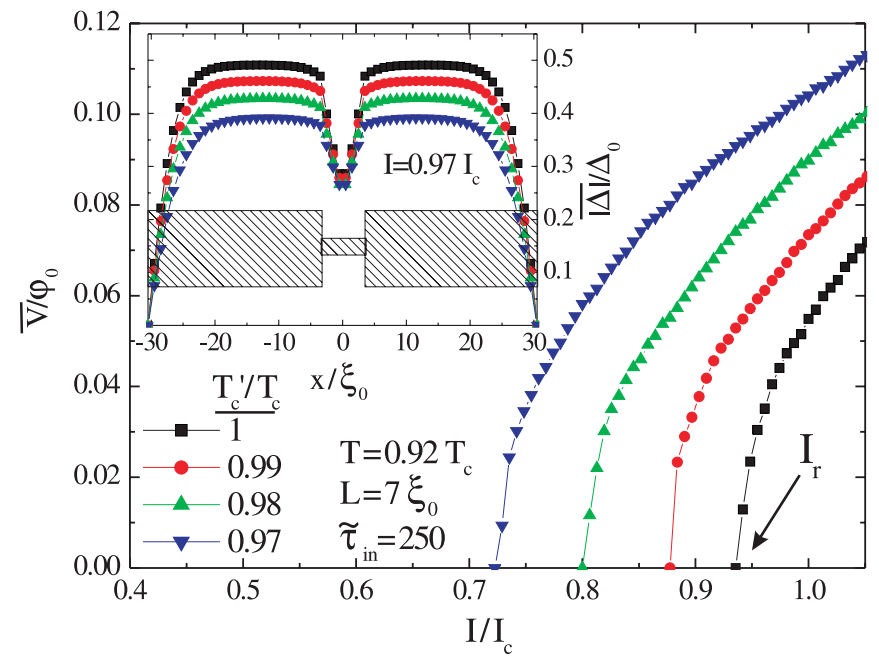

FIG. 5. (Color online) Current-voltage characteristics of the superconducting microbridge with length $L=7 \xi_{0} \simeq 1.7 \xi(T)$ calculated at $T=0.92 T_{c}$ and $\tilde{\tau}_{\text {in }}=250$. The inset shows the distribution of the time-averaged order parameter in the microbridge at different $T_{c}^{\prime}$. Current is normalized in units of the critical current of the microbridge with $T_{c}^{\prime}=T_{c}$.

For microbridges with length $L \gg L_{\text {in }}$ the relaxation of the "hot" quasiparticles occurs mainly in the microbridge and hence the effect of diffusion to the leads is rather weak. This is illustrated in Fig. 6 where we present $I-V$ curves for a relatively long microbridge $L=31 \xi_{0}\left(\simeq 0.6 L_{\text {in }}\right.$ at $\left.\tilde{\tau}_{\text {in }}=250\right)$. The change in the $I-V$ curves is much weaker in comparison with the shorter microbridge (compare with Fig. 3) and we found that for $L=51 \xi_{0} \simeq L_{\text {in }}$ the effect practically disappears for $\tilde{\tau}_{\text {in }}=250$.

There is one interesting effect when $L \lesssim L_{c}$. We find a decrease of $I_{r}$ and an increase of the resistance at $I \sim I_{r}$ but starting from some current $I>I_{r}$ the resistance of the

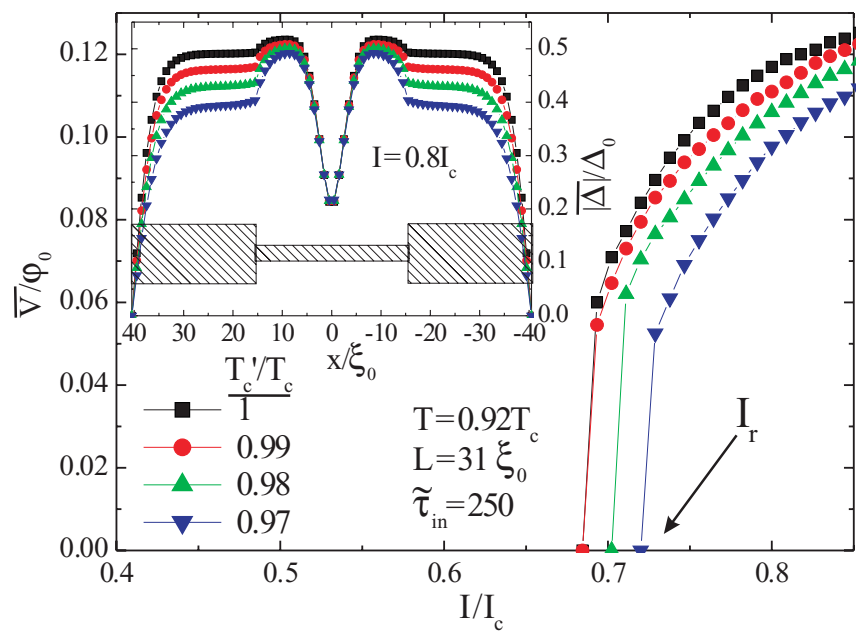

FIG. 6. (Color online) Current-voltage characteristics of the superconducting microbridge with length $L=31 \xi_{0} \simeq 7.3 \xi(T)$ calculated at $T=0.92 T_{c}$ and $\tilde{\tau}_{\text {in }}=250$. The inset shows the distribution of the time-averaged order parameter in the microbridge at different $T_{c}^{\prime}$. Current is normalized in units of the critical current of the microbridge with $T_{c}^{\prime}=T_{c}$.

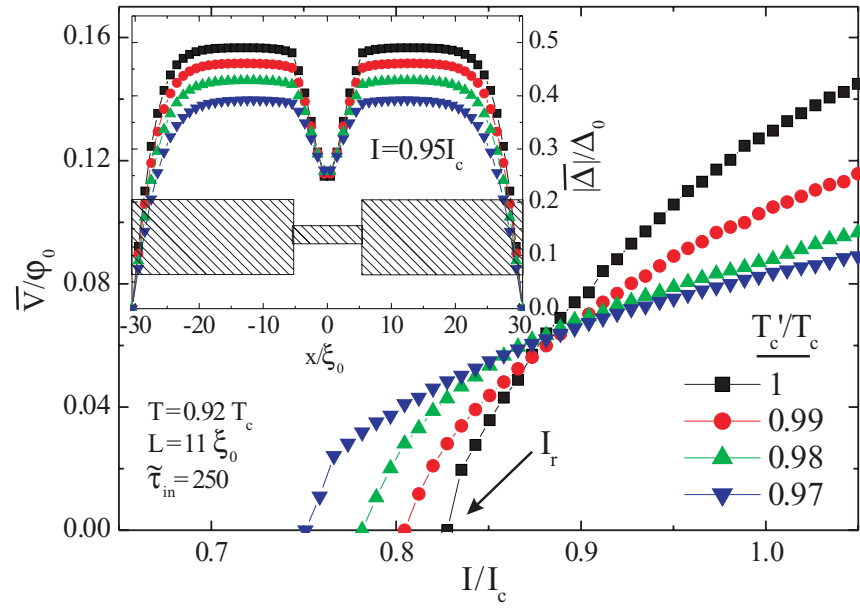

FIG. 7. (Color online) Current-voltage characteristics of the superconducting microbridge with length $L=11 \xi_{0} \simeq 2.6 \xi(T)$ calculated at $T=0.92 T_{c}$ and $\tilde{\tau}_{\text {in }}=250$. The inset shows the distribution of the time-averaged order parameter in the microbridge at different $T_{c}^{\prime}$. Current is normalized in units of the critical current of the microbridge with $T_{c}^{\prime}=T_{c}$.

microbridge decreases when $\Delta_{\text {lead }}$ is suppressed; see Fig. 7. At this length there is a competition between the influence of the order parameter in the leads and the "heating" of the quasiparticles on the $I-V$ curves. At low currents $I \sim I_{r}$ the value of $I_{r}$ is determined mainly by $\Delta_{\text {lead }}$ and $I_{r}$ decreases with decreasing $\Delta_{\text {leads }}$. At larger currents the "heating" of the quasiparticles becomes stronger because it increases with decreasing Josephson period. As a result decreasing $\Delta_{\text {lead }}$ weakens the "heating" effects and the voltage at fixed current decreases.

\section{DISCUSSION}

A typical feature of many experiments about the enhancement of the critical current and the negative magnetoresistance is the presence of finite resistance of the nanowires or microbridges even at low temperatures, ${ }^{3-7}$ which implies a strong influence of fluctuations. It can explain the absence of the hysteresis of $I-V$ curves observed in Refs. $3-7$. Indeed, it is known for example from the theory of Josephson junctions that fluctuations may completely destroy the hysteretic behavior. ${ }^{27}$ Therefore the current measured in those experiments is probably not $I_{c}$ but $I_{r}$. And indeed, the estimations made in Ref. 6 showed that the measured critical current was much smaller (more than 10 times) than the depairing current. Therefore we believe that our results can be directly applied for the explanation of the enhancement of the critical current found in Refs. 3-6. Taking into account that in zinc $L_{\text {in }} \simeq 25 \mu \mathrm{m}^{16}$ (when $\xi_{0} \simeq 250 \mathrm{~nm}^{6}$ ) it becomes clear why the effect was weak in a relatively long microbridge with $L=10 \mu \mathrm{m} \simeq 0.4 L_{\text {in }}$ and in short one with $L=1 \mu \mathrm{m} \simeq 4 \xi_{0} \cdot{ }^{6}$ In $\mathrm{Sn}$ the effect was absent for nanowires with $L=6-35 \mu \mathrm{m}^{4}$ because in tin $\xi_{0} \sim 55 \mathrm{~nm}$ and $L_{\text {in }} \simeq 470 \mathrm{~nm} .{ }^{16}$ The applicability of our result to the experiment of Rogachev et al. ${ }^{2}$ is questionable because those authors claimed that their critical current is about the depairing current and furthermore the experimental $I$ - $V$ curves were strongly hysteretic [see inset in Fig. 2(c) in Ref. 2]. 
Unfortunately no length dependence of the effect was studied in that work.

We believe that our result gives a clue in the understanding of the origin of the negative magnetoresistance at low currents $I \ll I_{r}$. It is believed that the finite resistance of the superconducting nanowires or microbridges at low currents originates from the finite rate of thermoactivated and/or quantum phase slips (see for example the review in Ref. 28). Each phase slip event is connected with one oscillation of the magnitude of the order parameter which provides the "heating" of the quasiparticles. Therefore, during a finite time $\min \left\{\tau_{\text {in }}, \tau_{\text {diff }} \sim L^{2} / D\right\}$ after the phase slip event the effective "temperature" of the quasiparticles will be larger than the bath temperature and the probability of the next phase slip becomes higher. It creates the condition for phase slip avalanches. By decreasing the order parameter in the leads one increases the flux of "hot" quasiparticles from the microbridge and therefore decreases the effective "temperature" and the probability of phase slip avalanches. The effect should be strongest in microbridges with $L \lesssim L_{\text {in }}$ where such a diffusion is the most effective one. In short microbridges with $L \lesssim 2 \xi(T)$ the suppression of the order parameter in the leads suppresses also $\Delta$ in the microbridge [due to the proximity effect; see Figs. 1(b) and 1(c)] and it gives the leading contribution to the probability for phase slips; i.e., it considerably increases. Therefore one should observe positive magnetoresistance in short microbridges. In MoGe $L_{\text {in }} \simeq 80 \mathrm{~nm}$ (when $\xi_{0} \simeq 13 \mathrm{~nm}$; see Ref. 25), in Nb $L_{\text {in }} \simeq 170 \mathrm{~nm}\left[\xi_{0}=28 \mathrm{~nm}\right.$ (Ref. 2)] in $\mathrm{Pb}$ $L_{\text {in }} \simeq 240 \mathrm{~nm}\left(\xi_{0}=40 \mathrm{~nm}\right)$, in $\operatorname{Al} L_{\text {in }} \simeq 8 \mu \mathrm{m}\left(\xi_{0}=140 \mathrm{~nm}\right)$, and in $\mathrm{Zn} L_{\text {in }} \simeq 25 \mu \mathrm{m}\left(\xi_{0}=250 \mathrm{~nm}\right)$. It correlates with the length of the nanowires or microbridges which were studied in Refs. 1-7 and where the negative magnetoresistance at low currents was observed both near and far from $T_{c}$.

From our theoretical calculations we found that the enhancement of $I_{r}$ is absent in the temperature interval $0.92<$ $T / T_{c}<0.99$ for $\tilde{\tau}_{\text {in }} \lesssim 20$ for all considered lengths of the microbridges $L=7-51 \xi_{0}$. This is not surprising because in this temperature interval the corresponding $L_{\text {in }}$ is about the coherence length and the period of oscillations of the order parameter is about $\tau_{\text {in }}$. For these conditions the effective "heating" is rather weak (see Ref. 24) and hence diffusion of "hot" quasiparticles does not play any role. For $\tilde{\tau}_{\text {in }}=60$ the effect is practically absent at $T / T_{c}>0.96$ [in this case $L_{\text {in }} \simeq$ $\left.7.5 \xi_{0} \sim \xi(T)\right]$ and it becomes noticeable at $T / T_{c}=0.92$ for microbridges with length $17 \xi_{0}<L<31 \xi_{0}$.
One more interesting effect which comes from our calculations is the nonmonotonous dependence of $I_{r}$ on the length of the microbridge at zero magnetic field (when $T_{c}^{\prime}=T_{c}$ ). One can see from comparing the values of $I_{r}$ in Figs. 3-7 that $I_{r}$ is minimal for $L=21 \xi_{0}$. The reason for such a behavior is the following. In a very long microbridge $L \gg L_{\text {in }}$ the "hot" quasiparticles, which can diffuse out the phase slip core, relax on the length scale $\sim L_{\text {in }}$ before they can reach the leads and $I_{r}$ does not depend on $L$. In a shorter bridge $L \lesssim L_{\text {in }}$ these "hot" quasiparticles are reflected from the leads and come back to the phase slip core providing increase of the local effective temperature in comparison with longer wire. As a result the retrapping current decreases with decreasing $L$ and reaches the minimal value at $L \lesssim 4 \xi$. For shorter wire the proximity effect from the leads starts to influence $I_{r}$, the main effect comes from the finite relaxation time of $|\Delta|,{ }^{24} I_{r}$ approaches $I_{c}$ (hysteresis shrinks), and both $I_{r}$ and $I_{c}$ rapidly grow with decreasing length of the microbridge.

\section{CONCLUSION}

The "heating" of quasiparticles, which occurs in the phase slip core due to oscillations of the order parameter, can be reduced by diffusion of the quasiparticles to the outside regions. It results in an enhancement of the retrapping current when one slightly suppresses the order parameter in the superconducting leads. The enhancement of $I_{r}$ strongly depends on the length of the microbridge: The effect is absent in short microbridges with length $L \lesssim 2 \xi(T)$ and it is weak in relatively long samples with length $L \gtrsim L_{\text {in }}$. Our results predict also a nonmonotonous dependence of the retrapping current $I_{r}$ on the length of the microbridge: It is minimal when $4 \xi(T) \lesssim L<L_{\text {in }}$. We should note that our results cannot be obtained in the framework of ordinary ${ }^{27}$ or extended ${ }^{20}$ time-dependent Ginzburg-Landau equations and one need to solve Eqs. (1)-(3) where nonlocal effects connected with a time delay of the response and the diffusion of nonequilibrium quasiparticles are taken into account.

\section{ACKNOWLEDGMENTS}

This work was supported by the Russian Foundation for Basic Research, Russian Agency of Education, under the Federal Target Programme "Scientific and Educational Personnel of Innovative Russia in 2009-2013" and the Flemish Science Foundation (FWO-Vl).

\footnotetext{
*vodolazov@ipm.sci-nnov.ru

${ }^{1}$ P. Xiong, A. V. Herzog, and R. C. Dynes, Phys. Rev. Lett. 78, 927 (1997).

${ }^{2}$ A. Rogachev, T.-C. Wei, D. Pekker, A. T. Bollinger, P. M. Goldbart, and A. Bezryadin, Phys. Rev. Lett. 97, 137001 (2006).

${ }^{3}$ M. L. Tian, N. Kumar, S. Y. Xu, J. G. Wang, J. S. Kurtz, and M. H. W. Chan, Phys. Rev. Lett. 95, 076802 (2005).

${ }^{4}$ M. Tian, N. Kumar, J. G. Wang, S. Y. Xu, and M. H. W. Chan, Phys. Rev. B 74, 014515 (2006).
}

${ }^{5}$ Yu. Chen, S. D. Snyder, and A. M. Goldman, Phys. Rev. Lett. 103, 127002 (2009).

${ }^{6}$ Yu. Chen, Y.-H. Lin, S. D. Snyder, and A. M. Goldman, Phys. Rev. B 83, 054505 (2011).

${ }^{7}$ M. Zgirski, K.-P. Riikonen, V. Touboltsev, and K. Yu. Arutyunov, Phys. Rev. B 77, 054508 (2008).

${ }^{8}$ In the discussion section we argue that the critical current measured in the majority of experiments (Refs. 3-7) is actually the retrapping current. The reason is finite resistance of the studied nanowires even at very low currents, which implies the strong influence of 
fluctuations leading to destruction of the hysteresis of currentvoltage characteristics. In a recent publication (Ref. 9) strong hysteretic behavior of aluminium nanowires was observed and the authors found that the retrapping current of a relatively short nanowire grows when one switches on the magnetic field.

${ }^{9}$ P. Li, P. M. Wu, Y. Bomze, I. V. Borzenets, G. Finkelstein, and A. M. Chang, Phys. Rev. B 84, 184508 (2011).

${ }^{10}$ D. A. Pesin and A. V. Andreev, Phys. Rev. Lett. 97, 117001 (2006).

${ }^{11}$ T. C. Wei, D. Pekker, A. Rogachev, A. Bezryadin, and P. M. Goldbart, Europhys. Lett. 75, 943 (2006).

${ }^{12} \mathrm{M}$. Yu. Kharitonov and M. V. Feigel'man, e-print arXiv:cond-mat/0612455.

${ }^{13}$ H. C. Fu, A. Seidel, J. Clarke, and D.-H. Lee, Phys. Rev. Lett. 96, 157005 (2006).

${ }^{14}$ D. Y. Vodolazov, Phys. Rev. B 75, 184517 (2007).

${ }^{15}$ K. Y. Arutyunov, Physica C 468, 272 (2008).

${ }^{16}$ M. Stuivinga, J. E. Mooij, and T. M. Klapwijk, J. Low Temp. Phys. 46, 555 (1982).

${ }^{17}$ D. Y. Vodolazov and F. M. Peeters, Phys. Rev. B 83, 224523 (2011).

${ }^{18}$ A. Schmid and G. Schön, J. Low Temp. Phys. 20, 207 (1975).

${ }^{19}$ A. I. Larkin and Yu. N. Ovchinnikov, Zh. Eksp. Teor. Fiz. 73, 299 (1977) [Sov. Phys. JETP 46, 155 (1977)].
${ }^{20}$ L. Kramer and R. J. Watts-Tobin, Phys. Rev. Lett. 40, 1041 (1978).

${ }^{21}$ R. J. Watts-Tobin, Y. Krähenbühl, and L. Kramer, J. Low Temp. Phys. 42, 459 (1981).

${ }^{22}$ H. Shibata, H. Takesue, T. Honjo, T. Akazaki, and Y. Tokura, Appl. Phys. Lett. 97, 212504 (2010).

${ }^{23}$ A. Anthore, H. Pothier, and D. Esteve, Phys. Rev. Lett. 90, 127001 (2003).

${ }^{24}$ D. Y. Vodolazov and F. M. Peeters, Phys. Rev. B 84, 094511 (2011).

${ }^{25}$ M. Liang and M. N. Kunchur, Phys. Rev. B 82, 144517 (2010).

${ }^{26} \mathrm{We}$ did not present results for smaller values of $T_{c}^{\prime}$ because in this case the order parameter is strongly suppressed near the ends of the microbridge and the order parameter first starts to oscillate there. This behavior is out of interest in this paper because such oscillations create asymmetry in the system with respect to the center of the microbridge and our numerical method should be seriously modified and numerically extended to study such a processes correctly.

${ }^{27}$ M. Tinkham, Introduction to Superconductivity (McGraw-Hill, New York, 1996).

${ }^{28}$ K. Yu. Arutyunov, D. S. Golubov, and A. D. Zaikin, Phys. Rep. 464, 1 (2008). 